\title{
Antioxidative activities of chromatographic fractions obtained from root, fruit and leaf of Mengkudu (Morinda citrifolia L.)
}

\begin{abstract}
Crude extracts of root, leaf and fruit of Morinda citrifolia were fractionated on a Sephadex LH-20 column with ethanol as eluate. Based on UV absorption intensity of phenolic compound $(725 \mathrm{~mm})$ the Sephadex LH-20 column was able to separate fruit, leaf and root extracts into six, five and five fractions, respectively. The results showed that all the fractions tested exhibited considerably high antioxidative activity in the ferric thiocyanate assay and thiobarbituric acid test and the activities of some of the fractions were as good as those of either tocopherol or BHT. The fractions from different parts of the plants were found to contain different amounts of total phenolic compounds, which, interestingly, do not correspond to the antioxidative activity measured. This is probably due to the presence of different phenolics in the samples, with different antioxidative activities which involves various mechanisms inhibiting the oxidation process. The study suggested that root, leaf and fruit of M. citrifolia might contribute significantly to exogenous antioxidant which is crucial in combating oxidative stress.
\end{abstract}

Keyword: Morinda citrifolia, Antioxidative activity, Chromatographic fractions 